PALEO

Revue d'archéologie préhistorique

$23 \mid 2012$

Varia

\title{
Ours, hommes, hyènes : qui a occupé la grotte de Bourdette (Sainte-Colombe-en-Bruilhois, Lot-et-Garonne, France)?
}

Emmanuel Discamps, Myriam Boudadi-Maligne, Jean Chagneau, Dominique Armand, Jean-Luc Guadelli et Michel Lenoir

\section{(2) OpenEdition}

Journals

Édition électronique

URL : http://journals.openedition.org/paleo/2320

DOI : $10.4000 /$ paleo.2320

ISSN : 2101-0420

Éditeur

SAMRA

Édition imprimée

Date de publication : 15 décembre 2012

Pagination : 117-136

ISSN : 1145-3370

\section{Référence électronique}

Emmanuel Discamps, Myriam Boudadi-Maligne, Jean Chagneau, Dominique Armand, Jean-Luc Guadelli et Michel Lenoir, «Ours, hommes, hyènes : qui a occupé la grotte de Bourdette (SainteColombe-en-Bruilhois, Lot-et-Garonne, France) ? », PALEO [En ligne], 23 | 2012, mis en ligne le 19 mars 2013, consulté le 25 juillet 2020. URL : http://journals.openedition.org/paleo/2320 ; DOI : https:// doi.org/10.4000/paleo.2320

Ce document a été généré automatiquement le 25 juillet 2020.

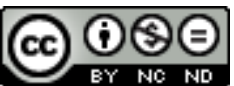

PALEO est mis à disposition selon les termes de la licence Creative Commons Attribution - Pas d'Utilisation Commerciale - Pas de Modification 4.0 International. 


\title{
Ours, hommes, hyènes : qui a occupé la grotte de Bourdette (Sainte-Colombe-en-Bruilhois, Lot-et-Garonne, France)?
}

\author{
Emmanuel Discamps, Myriam Boudadi-Maligne, Jean Chagneau, \\ Dominique Armand, Jean-Luc Guadelli et Michel Lenoir
}

\section{1 - Présentation et historique du site de Bourdette}

1 La grotte de Bourdette est située sur la commune de Sainte-Colombe-en-Bruilhois (Lotet-Garonne) à environ $10 \mathrm{~km}$ à l'ouest d'Agen (fig. 1A). À l'occasion de travaux d'exploitation d'une carrière de calcaire, un réseau karstique complexe fut mis au jour dans la falaise, à sept mètres du niveau du sol. Des opérations de fouilles de sauvetage ont été menées par Jean Chagneau, entre 1976 et 1981, sur trois locus principaux ayant livré des dépôts fossilifères quaternaires : la galerie I (SCB I), la galerie II (SCB II) et la salle du cheval (SDC ; fig. 1B).

Les observations sédimentologiques faites sur les deux locus principaux (galeries I et II) identifient au sein du remplissage sédimentaire neuf couches pour SCB 1 et 24 pour SCB 2 (Chagneau 1980, 1982 ; Le Tensorer 1979, 1981). Dans la première galerie, la couche 8, près de la base de la séquence, concentre la majorité des restes paléontologiques, et n'est surmontée que par des couches où les vestiges sont plus rares (couches 1 à 7). Dans la deuxième galerie, seules trois couches fossilifères (de haut en bas : couches 6, 7 et 8) ont été distinguées au sein d'une séquence d'environ 4,5 mètres de puissance. La chronologie de ces dépôts est restée incertaine, étant tour à tour attribués au «Würm 1 » (Chagneau 1980; Le Tensorer 1981) ou à l' «interstade Würm II/III » (Chagneau 1982) selon les auteurs. Le site étant victime de l'action répétée de fouilleurs clandestins qui nuisent au bon déroulement des opérations, une partie des vestiges ne 
peut être récoltée dans sa position stratigraphique originelle, bien que l'existence de différents niveaux semble attestée.

3 Les nombreux restes d'Ours mis au jour (environ 7520 restes soit près de $95 \%$ des vestiges fauniques, avec un NMI de 126 pour la seule galerie II et un nombre important de juvéniles) et la présence de bauges et de griffades sur les parois plaident en faveur d'une fonction principale du site en tant que tanière d'Ours. En outre, le site livre également les restes d'une vingtaine d'espèces d'Ongulés, de Carnivores et de petits Vertébrés, ainsi que quelques éléments lithiques (Chagneau 1980, 1982).

L'association d'une telle gamme de vestiges paraît, a priori, peu conciliable avec une fonction unique de la grotte en tant que tanière d'Ours. Ce Carnivore n'est effectivement pas connu pour accumuler des restes d'autres espèces dans les cavités qu'il fréquente (Stiner 1997). Les ensembles osseux et lithiques de Bourdette résultentils d'un palimpseste de différentes occupations? Le cas échéant, est-il possible de les distinguer, de les documenter et d'établir leur chronologie?

5 La réponse à ces questions, récurrentes en contexte karstique (p.ex. Brugal et Jaubert 1991 ; Stiner 1997 ; Villa et Soressi 2000 ; Discamps 2011 ; Discamps et al. sous presse), constitue un préalable essentiel à une meilleure compréhension de la nature des fréquentations de la grotte de Bourdette.

\section{2 - Méthodes d'analyses}

6 Afin de mieux distinguer les différentes occupations de Bourdette et d'en établir la chronologie, nous avons privilégié une approche interdisciplinaire incluant l'étude taphonomique des restes osseux, l'analyse typotechnologique des pièces lithiques et la confrontation des différents éléments de chronologie disponibles (biochronologie, datations radiocarbone, attribution chronoculturelle des industries lithiques).

\section{1 - Taphonomie osseuse}

$7 \quad$ L'étude taphonomique des vestiges osseux permet de fournir des clés interprétatives utiles afin de comprendre l'origine d'espèces inattendues dans un contexte de tanière d'Ours. Les travaux précédents s'étant concentrés sur les restes d'Ours (Chagneau 1980, 1982), l'ensemble des restes osseux hors Ours a été analysé dans une perspective taphonomique par deux d'entre nous (ED et MBM). L'ensemble du matériel qui ne pouvait être attribué spécifiquement à l'Ours des cavernes ou à l'Ours brun a été isolé, puis chaque fragment a été déterminé et observé à la loupe (grossissement x10). Les diverses atteintes taphonomiques abiotiques (émoussés, concrétions, traces de manganèse, fissures longitudinales, stries de piétinement, dissolution et corrosion) ont été notées tout comme la présence ou l'absence de traces d'action anthropique ou des Carnivores (os rongés, digérés). Les restes non déterminés spécifiquement n'ont pas été inclus dans la base de données taphonomique, la grande majorité d'entre eux appartenant très certainement à l'Ours des cavernes (ce dernier représente près de $95 \%$ des restes fauniques). La base de données ainsi constituée inclut un total de 542 restes déterminés spécifiquement (tabl. 1).

8 Au-delà des considérations pratiques de la méthodologie d'analyse, il parait également opportun de réfléchir sur la pertinence des critères à employer pour distinguer 
différents agents accumulateurs en taphonomie osseuse. Ce type de débats, relativement anciens (cf. Fosse 1995), a été nourri par une large littérature ces deux dernières décennies (p.ex. Cruz-Uribe 1991 ; Fosse 1994, 1995, 1997 ; Brugal et al. 1997 ; Fosse et al. 1998 ; Pickering 2002 ; Letourneux 2003; Marra et al. 2004; Villa et al. 2004 ; Costamagno et al. 2005 ; Coumont 2006, 2009; Faith et Behrensmeyer 2006; Pokines et Kerbis Peterhans 2007 ; Egeland et al. 2008 ; Beauval et Morin 2010 ; Kuhn et al. 2010).

9 Sur la base des travaux de ces auteurs, il est possible de résumer en un tableau (tabl. 2) les critères les plus utiles pour distinguer différents types d'accumulations, en considérant trois fonctions de sites "caricaturaux » (dans le sens où elles n'intègrent pas toute la variabilité des accumulations possibles) : les sites anthropiques, les repaires d'Hyène et les avens-pièges (se reporter à Coumont 2006 ou Discamps 2011 pour une discussion plus détaillée, critère par critère). Ces critères sont pour l'essentiel des marqueurs de l'activité des hyènes (abondance de l'Hyène, des hyénons, des coprolithes, d'os rongés et digérés, de cylindres diaphysaires, morphotypes osseux) aux côtés d'autres, souvent plus fugaces (traces anthropiques) ou plus délicats à discuter (degré de complétude des os longs, connexions anatomiques). Ce sont ces critères qui ont été privilégiés dans ce travail, au détriment d'autres beaucoup moins fiables (p.ex. les profils squelettiques, critère pour lequel les méthodes de récolte ou d'analyse peuvent biaiser les interprétations de manière non négligeable; cf. Marean et Kim 1998).

10 Il est intéressant de noter que l'observation de trois des critères retenus (os rongés, restes digérés et traces anthropiques) dépend pour grande partie de la conservation des surfaces corticales. En conséquence, lors de l'étude taphonomique, un champ supplémentaire dit de "degré de lisibilité des surfaces corticales » a également été enregistré dans la base de données (comme préconisé par exemple par Mallye 2007 ou Rendu 2010). Cet indicateur est utile pour estimer si, par exemple, l'absence de stries de découpe peut être induite par la rareté des surfaces corticales bien préservées. 
Figure 1 - A) Localisation de la grotte de Bourdette (fonds de carte (c) Géoatlas). B) Plan général de la partie terminale du réseau karstique montrant les différents locus (galerie I, galerie II et salle du cheval SDC) et l'extension approximative des zones fouillées (en gris clair).
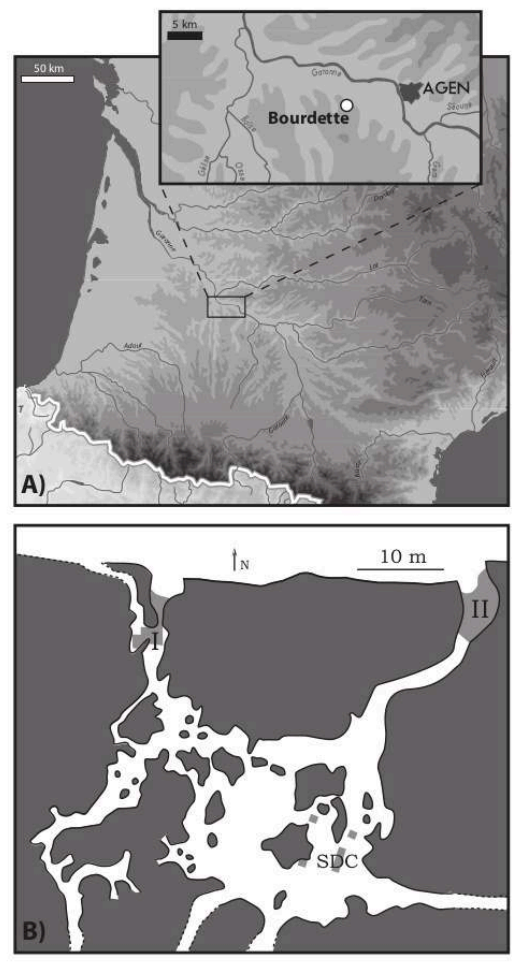

Tableau 1 - Liste faunique des restes déterminés, par locus et couche (HS : hors stratigraphie ; 1 : pièces hors stratigraphie et locus inconnu ; 2 : Ours exclu).

\begin{tabular}{|c|c|c|c|c|c|c|c|c|c|c|c|c|c|}
\cline { 2 - 15 } \multicolumn{1}{c|}{} & \multicolumn{9}{c|}{ Galerie I } & \multicolumn{5}{c|}{ Galerie II } & \multirow{2}{*}{ SDC } & \multirow{2}{*}{ HS $^{\mathbf{1}}$} & \multirow{2}{*}{ Total } \\
\cline { 2 - 15 } & c. 1 & c. $\mathbf{3}$ & c. $\mathbf{6}$ & c. $\mathbf{7}$ & c. $\mathbf{8}$ & HS & c. $\mathbf{6}$ & c. $\mathbf{7}$ & c. $\mathbf{8}$ & HS & & & \\
\hline Hyène & 3 & 1 & & 1 & 11 & 3 & 11 & 59 & 26 & 8 & 2 & 5 & 130 \\
\hline Lion & & & & & 1 & & & & & & & & 1 \\
\hline Loup & & & & & & & & 3 & 2 & 1 & & & 6 \\
\hline Lynx & & & & & & & & & & 1 & & & 1 \\
\hline Blaireau & & & & & & & 12 & & & & & & 12 \\
\hline Putois & & & & & & & & 1 & & & & & 1 \\
\hline Vulpinés & & & & & & & 1 & 2 & & & & & 3 \\
\hline Carnivores $^{2}$ & $\mathbf{3}$ & $\mathbf{1}$ & $\mathbf{0}$ & $\mathbf{1}$ & $\mathbf{1 2}$ & $\mathbf{3}$ & $\mathbf{2 4}$ & $\mathbf{6 5}$ & $\mathbf{2 8}$ & $\mathbf{1 0}$ & $\mathbf{2}$ & $\mathbf{5}$ & $\mathbf{1 5 4}$ \\
\hline
\end{tabular}

\begin{tabular}{|c|c|c|c|c|c|c|c|c|c|c|c|c|c|}
\hline Bovinés & & 5 & 1 & & 52 & 4 & 7 & 14 & 19 & 5 & 2 & 12 & 121 \\
\hline Cheval & 1 & 5 & 1 & 1 & 50 & 14 & 16 & 38 & 32 & 4 & 31 & 22 & 215 \\
\hline Cerf & & & & & 1 & 1 & & & & & & & 2 \\
\hline Hydruntin & & & & & 3 & 1 & & 2 & 2 & & & & 8 \\
\hline Mammouth & & & & & & & & & 1 & & & & 1 \\
\hline Mégacéros & & & & 1 & 1 & & 1 & & 2 & 1 & 1 & & 7 \\
\hline Renne & & & & 1 & & 3 & 10 & 2 & 2 & & 1 & 19 \\
\hline Rhinocéros & & & & & & & & 5 & & & & & 5 \\
\hline Sanglier & & & & & 2 & & & 1 & 1 & & & & 4 \\
\hline Ongulés & $\mathbf{1}$ & $\mathbf{1 0}$ & $\mathbf{2}$ & $\mathbf{2}$ & $\mathbf{1 1 0}$ & $\mathbf{2 0}$ & $\mathbf{2 7}$ & $\mathbf{7 0}$ & $\mathbf{5 9}$ & $\mathbf{1 2}$ & $\mathbf{3 4}$ & $\mathbf{3 5}$ & $\mathbf{3 8 2}$ \\
\hline
\end{tabular}

\begin{tabular}{|c|l|l|l|l|l|l|l|l|l|l|l|l|l|}
\hline Lièvre & & & & & & & & & & & 1 & & 1 \\
\hline Taupe & & & & & & & & & 1 & & & & 1 \\
\hline Rapace & & & & & & & & 2 & & & & & 2 \\
\hline Microfaune & & & & & & & & 1 & & & & 1 & 2 \\
\hline $\begin{array}{c}\text { Petits } \\
\text { vertébrés }\end{array}$ & & & & & & & $\mathbf{3}$ & $\mathbf{1}$ & & $\mathbf{1}$ & $\mathbf{1}$ & $\mathbf{6}$ \\
\hline
\end{tabular}


Tableau 2 - Critères taphonomiques de distinction des agents accumulateurs consensuels, selon quatre archétypes de fonction de site (cf. références bibliographiques et discussion dans le texte).

\begin{tabular}{|c|c|c|c|c|}
\hline & \multirow{2}{*}{$\begin{array}{l}\text { "Repaire " } \\
\text { d'hyènes }\end{array}$} & \multicolumn{2}{|c|}{$\begin{array}{l}\text { "Aven-piège " où l'action } \\
\text { de l'Hyène est }\end{array}$} & \multirow{2}{*}{$\begin{array}{l}\text { Site } \\
\text { " anthropique " }\end{array}$} \\
\hline & & importante & faible & \\
\hline $\begin{array}{l}\text { Présence des } \\
\text { carnivores }\end{array}$ & \multicolumn{2}{|c|}{ Forte } & \multicolumn{2}{|c|}{ Faible } \\
\hline $\begin{array}{l}\text { Spectre des } \\
\text { carnivores }\end{array}$ & $\begin{array}{l}\text { Hyènes } \\
\text { dominent }\end{array}$ & \multicolumn{3}{|c|}{ Variable } \\
\hline $\begin{array}{l}\text { Profil de mortalité } \\
\text { des hyènes }\end{array}$ & $\begin{array}{l}\text { Juvéniles } \\
\text { importants }\end{array}$ & \multicolumn{3}{|c|}{ Adultes dominent } \\
\hline Os rongés & \multicolumn{2}{|c|}{ Nombreux } & \multicolumn{2}{|c|}{ Rares voire absents } \\
\hline $\begin{array}{l}\text { Os et dents } \\
\text { régurgités }\end{array}$ & \multicolumn{2}{|c|}{$\begin{array}{l}\text { - Nombreux } \\
\text { - Fragments de taille } \\
\text { importante possibles }\end{array}$} & \multicolumn{2}{|c|}{ Rares voire absents } \\
\hline $\begin{array}{c}\text { Traces } \\
\text { anthropiques }\end{array}$ & \multicolumn{3}{|c|}{ Rares voire absentes } & Nombreuses \\
\hline $\begin{array}{l}\text { Coprolithes (pour } \\
\text { l'Hyène, souvent } \\
\text { gros, ronds et bien } \\
\text { minéralisés) }\end{array}$ & $\begin{array}{c}\text { - Nombreux } \\
\text { et } \\
\text { concentrés } \\
\text { - Zonation } \\
\text { (latrines) }\end{array}$ & Rares & \multicolumn{2}{|c|}{ Rares voire absents } \\
\hline Os longs entiers & \multicolumn{2}{|c|}{$\begin{array}{l}\text { Oui, surtout pour les } \\
\text { métapodes }\end{array}$} & $\begin{array}{l}\text { Oui, pour } \\
\text { tous les os } \\
\text { longs }\end{array}$ & $\begin{array}{l}\text { Rares voire } \\
\text { absents }\end{array}$ \\
\hline $\begin{array}{l}\text { Connexions } \\
\text { anatomiques }\end{array}$ & Rares & \multicolumn{2}{|c|}{$\begin{array}{l}\text { - Abondance variable } \\
\text { - Squelettes entiers } \\
\text { possibles }\end{array}$} & $\begin{array}{l}\text { Rares voire } \\
\text { absentes }\end{array}$ \\
\hline $\begin{array}{l}\text { Cylindres } \\
\text { diaphysaires }\end{array}$ & \multicolumn{2}{|c|}{ Nombreux } & \multicolumn{2}{|c|}{ Rares voire absents } \\
\hline $\begin{array}{l}\text { Morphotypes } \\
\text { osseux typiques } \\
\text { de l'action de } \\
\text { l'Hyène }\end{array}$ & \multicolumn{2}{|c|}{ Nombreux } & \multicolumn{2}{|c|}{ Rares voire absents } \\
\hline
\end{tabular}

\section{2 - Biochronologie}

11 Afin de discuter de l'âge des faunes de Bourdette, des analyses morphométriques ont été menées sur l'Ours, les Equidés, l'Hyène et le Loup. Pour l'Ours, les analyses ont porté sur la morphologie de l'entoconide des carnassières inférieures telle qu'elle est définie par la typologie d'A. Argant (1995). Le calcul des indices morphodynamiques (Rabeder 1991) n'a pas été utilisé car peu applicable en dehors de l'arc alpin. En effet, devant les résultats particuliers obtenus dans le site de "Caverna generosa », où les niveaux les plus récents ont un index morphodynamique plus petit que les plus anciens, F. Bona (2004) émet plusieurs hypothèses et en particulier que la méthode ne serait pas applicable à la zone étudiée. À Bourdette, le calcul de ces indices aboutit à des valeurs aberrantes $(252,9$ pour les quatrièmes prémolaires supérieures et 275 pour les inférieures), très nettement supérieures à l'ensemble des gisements de comparaison de G. Rabeder (1991).

12 Pour les autres espèces, les données biométriques ont été analysées afin de discuter du stade évolutif des taxons considérés en référence aux travaux menés dans le sud-ouest de la France (p.ex. Prat 1968 ; Guadelli 1987 ; Boudadi-Maligne 2010). 


\section{3 - Taphonomie osseuse : quel(s) agent(s) accumulateur(s)?}

\section{1 - Présence de l'Hyène}

13 Lorsque l'Ours est exclu du spectre faunique, les Ongulés dominent nettement la faune de Bourdette (70\% du NRD; tabl. 1). L'Hyène est présente dans toutes les couches et dans tous les locus de Bourdette, à l'exception de la couche 6 de SCB I. Avec un total de 130 restes, elle est le deuxième Carnivore le plus représenté après l'Ours, et représente un minimum de dix individus (NMI de combinaison) dont, fait remarquable, trois juvéniles (fig. 2B).

14 En dehors des restes squelettiques, la présence de l'Hyène est également attestée par l'abondance de coprolithes $(n=174)$ qui présentent une morphologie typique : ils sont ronds, bien minéralisés, pluricentimétriques, et contiennent parfois des fragments osseux digérés (fig. 2A). Si pour la majorité des coprolithes, les informations sur leur provenance stratigraphique manquent, leur présence est néanmoins avérée dans toutes les couches et locus, à l'exception de la couche 7 du locus I (tabl. 3).

Tableau 3 - Répartition des coprolithes par locus et couche (HS : hors stratigraphie ; 1 : pièces hors stratigraphie et locus inconnu).

\begin{tabular}{|l|c|c|c|c|c|c|c|c|c|c|c|c|c|}
\cline { 2 - 14 } \multicolumn{1}{c|}{} & \multicolumn{4}{c|}{ Galerie I } & \multicolumn{4}{c|}{ Galerie II } & \multirow{2}{*}{ SDC } & HS & Total \\
\cline { 2 - 14 } & c. $\mathbf{1}$ & c. $\mathbf{3}$ & c. $\mathbf{6}$ & c. $\mathbf{7}$ & c. $\mathbf{8}$ & HS & c. $\mathbf{6}$ & c. $\mathbf{7}$ & c. $\mathbf{8}$ & HS & & & \\
\hline Coprolithes & 4 & 2 & 1 & & 1 & 13 & 5 & 10 & 14 & & 3 & 121 & 174 \\
\hline
\end{tabular}


Tableau 4 - Abondance des os émoussés et rongés par locus, couche et taxon (nombre de restes total et pourcentages par catégorie de restes affectés).

\begin{tabular}{|c|c|c|c|c|c|}
\hline & & $\begin{array}{c}\text { Tous } \\
\text { taxons }\end{array}$ & Bovinés & Cheval & Hyène \\
\hline \multirow{6}{*}{$\begin{array}{l}\frac{n}{3} \\
\frac{0}{0} \\
\stackrel{0}{0}\end{array}$} & NR & 548 & 121 & 215 & 130 \\
\hline & Abrasion & $64 \%$ & $85 \%$ & $50 \%$ & $70 \%$ \\
\hline & stade 1 & $34 \%$ & $35 \%$ & $26 \%$ & $55 \%$ \\
\hline & stade 2 & $22 \%$ & $35 \%$ & $18 \%$ & $13 \%$ \\
\hline & stade 3 & $8 \%$ & $15 \%$ & $6 \%$ & $2 \%$ \\
\hline & Os rongés & $4 \%$ & $3 \%$ & $2 \%$ & $2 \%$ \\
\hline
\end{tabular}

\begin{tabular}{|c|c|c|c|c|c|}
\hline \multirow{6}{*}{$\frac{\stackrel{\infty}{\dot{0}}}{\stackrel{-}{\dot{B}}}$} & NR & 125 & 52 & 50 & 11 \\
\hline & Abrasion & $62 \%$ & $90 \%$ & $42 \%$ & $18 \%$ \\
\hline & stade 1 & $36 \%$ & $47 \%$ & $32 \%$ & $0 \%$ \\
\hline & stade 2 & $19 \%$ & $27 \%$ & $10 \%$ & $18 \%$ \\
\hline & stade 3 & $7 \%$ & $16 \%$ & $0 \%$ & $0 \%$ \\
\hline & Os rongés & $3 \%$ & $2 \%$ & $0 \%$ & $0 \%$ \\
\hline
\end{tabular}

\begin{tabular}{|c|c|c|c|c|c|}
\hline \multirow{6}{*}{$\begin{array}{l}\dot{0} \\
\stackrel{y}{=} \\
\dot{0}\end{array}$} & NR & 51 & 7 & 16 & 11 \\
\hline & Abrasion & $33 \%$ & $67 \%$ & $34 \%$ & $45 \%$ \\
\hline & stade 1 & $21 \%$ & $17 \%$ & $27 \%$ & $27 \%$ \\
\hline & stade 2 & $6 \%$ & $33 \%$ & $7 \%$ & $0 \%$ \\
\hline & stade 3 & $6 \%$ & $17 \%$ & $0 \%$ & $18 \%$ \\
\hline & Os rongés & $0 \%$ & $0 \%$ & $0 \%$ & $0 \%$ \\
\hline
\end{tabular}

\begin{tabular}{|c|c|c|c|c|c|}
\hline \multirow{6}{*}{$\begin{array}{l}\hat{u} \\
\stackrel{=}{=} \\
\dot{J}\end{array}$} & NR & 137 & 14 & 38 & 59 \\
\hline & Abrasion & $88 \%$ & $92 \%$ & $82 \%$ & $89 \%$ \\
\hline & stade 1 & $50 \%$ & $23 \%$ & $34 \%$ & $81 \%$ \\
\hline & stade 2 & $29 \%$ & $46 \%$ & $40 \%$ & $8 \%$ \\
\hline & stade 3 & $9 \%$ & $23 \%$ & $8 \%$ & $0 \%$ \\
\hline & Os rongés & $4 \%$ & $0 \%$ & $0 \%$ & $2 \%$ \\
\hline
\end{tabular}

\begin{tabular}{|c|c|c|c|c|c|}
\hline \multirow{6}{*}{ 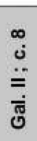 } & NR & 88 & 19 & 32 & 26 \\
\hline & Abrasion & $77 \%$ & $84 \%$ & $74 \%$ & $76 \%$ \\
\hline & stade 1 & $35 \%$ & $15 \%$ & $32 \%$ & $57 \%$ \\
\hline & stade 2 & $29 \%$ & $54 \%$ & $26 \%$ & $19 \%$ \\
\hline & stade 3 & $13 \%$ & $15 \%$ & $16 \%$ & $0 \%$ \\
\hline & Os rongés & $4 \%$ & $0 \%$ & $3 \%$ & $0 \%$ \\
\hline
\end{tabular}

Figure 2 - Exemples de témoignage de l'occupation de la grotte de Bourdette par l'Hyène. A) Coprolithes d'hyènes, dont certains contiennent des fragments d'os digérés (en médaillon). $B$ ) Restes d'hyénons.
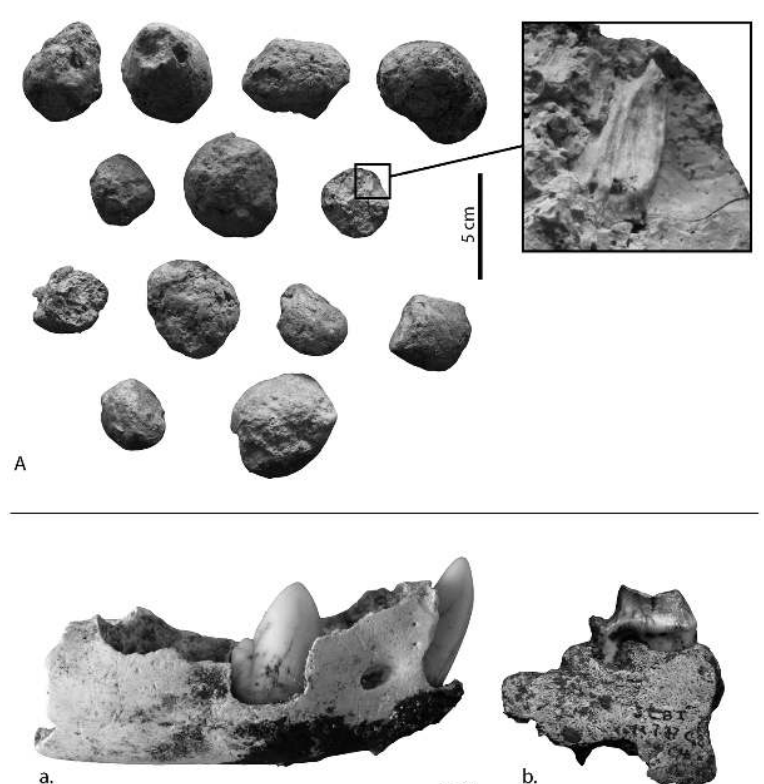

$1 \mathrm{~cm}$
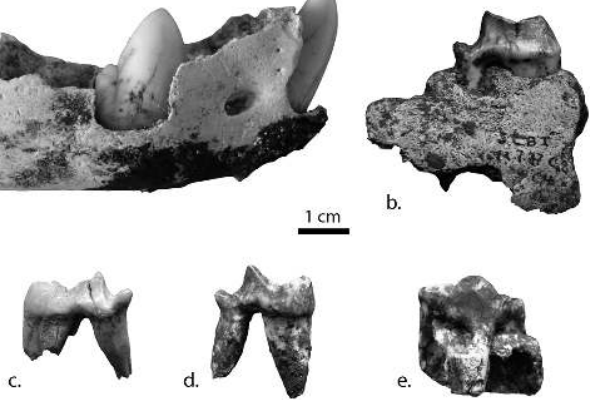


\section{2 - Atteintes taphonomiques}

15 La lecture taphonomique des pièces est particulièrement délicate à Bourdette car l'action de nombreux processus climato-édaphiques a réduit considérablement la lisibilité des surfaces corticales. Sur l'ensemble du matériel (Ours exclu), $18 \%$ des restes sont concrétionnés, et les attaques chimiques sont fréquentes : parfois légères (30\% des restes présentent des cupules de dissolution) mais souvent importantes ( $47 \%$ des restes sont corrodés). Cependant, l'atteinte taphonomique la plus fréquente est l'abrasion: $64 \%$ des restes présentent des surfaces polies et/ou des bords émoussés. La fréquence des pièces atteintes tout comme l'intensité de l'abrasion (codée de 1 à 3 , de la plus faible à la plus intense) varient selon les couches, locus et taxons (tabl. 4). Seule la couche 6 du locus II semble se différencier des autres ensembles (avec deux fois moins d'émoussés), mais la faiblesse des échantillons empêche toute interprétation statistiquement fiable de ces différences. Il n'en reste pas moins que les pièces abrasées sont fréquentes dans tous les locus, couches et pour tous les taxons.

Un tel degré d'abrasion (surfaces polies, bords émoussés) peut être causé par de nombreux processus naturels, souvent difficiles à distinguer (Costamagno 1999): activité éolienne (Shipman et Rose 1983), piétinement (Brain 1967), processus fluviatiles (Voorhies 1969; Andrews 1995; Behrensmeyer 1990; Fernandez-Jalvo et Andrews 2003), action des Gastéropodes (Morel 1986) et charriage à sec (Koby 1943, 1953). Ce dernier processus est bien connu pour les tanières d'Ours : "avant d'être enterrés les ossements sont déplacés et charriés à d'innombrables reprises, de sorte que,suivant la nature du sol de la caverne, ils se polissent plus ou moins » (Koby 1953). L'abondance des os émoussés à Bourdette pourrait donc s'expliquer par la circulation d'ours dans la cavité, même si d'autres processus, comme le ruissellement, ne peuvent être exclus.

Ces différentes atteintes abiotiques, abrasion en tête, complexifient l'identification des traces de consommation des ossements par l'Homme ou les Carnivores. Aucune trace de découpe n'a pu être identifiée sur le matériel. Des traces de Carnivores sont documentées pour presque tous les taxons (Bovinés, Cheval, Cerf, Mégacéros, Mammouth, Renne, Rhinocéros, Hyène et Lion), mais elles sont rares : 4 \% des restes présentent des traces de manducation (os rongés). Ce pourcentage varie légèrement selon les locus, couches et taxons, le plus souvent entre 2 et $4 \%$ des restes, rarement en dessous, sauf quand les effectifs sont très faibles (tabl. 4). Si l'on exclut les dents des décomptes, $9 \%$ des ossements sont rongés. Les restes digérés représentent eux $5 \%$ du matériel faunique. Ces pourcentages sont relativement faibles comparativement à ce qui est connu dans d'autres repaires d'Hyène où les os rongés représentent souvent environ 15 à 50 \% des restes (Fosse 1994 ; Letourneux 2003 ; Marra et al. 2004 ; Bourdillat 2008; Fourvel 2008; Beauval et Morin 2010; Discamps 2011). Aux Rochers de Villeneuve, seuls 2 à $3 \%$ des restes portent des traces d'action des dents de carnivores, mais ce faible pourcentage s'explique par la prise en compte de petits fragments, très abondants : les restes inférieurs à $5 \mathrm{~cm}$ de long, rarement rongés, représentent 81 à $88 \%$ du matériel (Beauval et Morin 2010). À Bourdette, seuls $47 \%$ des restes sont inférieurs à $5 \mathrm{~cm}$ de long et, dans ces décomptes, seuls les restes déterminés sont considérés (cf. 2.1) : la rareté des os rongés ne peut donc être expliquée par ce paramètre dimensionnel. Elle est plus vraisemblablement due à l'importance des os émoussés : seuls $57 \%$ des surfaces corticales ont été jugées comme potentiellement lisibles. 
Ce type de traces (os rongés, digérés) laisse à penser qu'une partie au moins des restes d'Herbivores a été consommée par un Carnivore. Même si une large gamme de Carnivores est connue pour rogner des ossements, dont le Loup (Binford 1981; Campmas et Beauval 2008), l'Ours (Perez-Rama et al. 2010; Saladié et al. 2011) et les grands félins (de Ruiter et Berger 2000 ; Stiner et al. 2012), l'intensité des attaques à Bourdette suggère que ces traces sont plus vraisemblablement imputables à l'Hyène. Pour exemple, un humérus de Rhinocéros a été découvert à Bourdette attaqué à ses deux extrémités de façon caractéristique (fig. 3) : ce morphotype de destruction est notamment décrit pour le repaire d'Hyène de Camiac (Discamps 2011).

\section{3 - Autres éléments}

19 À Bourdette, $53 \%$ des ossements sont complets ou presque complets. Ces pourcentages varient selon les locus, couches, taxons, mais restent proches tout en restant relativement élevés (tabl. 5). Il est cependant important de souligner que la répartition squelettique de ces os complets est particulière : pour les Ongulés, seuls des éléments de l'autopode sont complets (carpiens, tarsiens, métapodes, phalanges, sésamoïdes), à l'exception d'une patella complète de Cheval, et les éléments du stylopode et zeugopode (os longs hors métapodes) sont systématiquement fracturés. Pour l'Hyène, à l'inverse, un tibia, un fémur et plusieurs vertèbres sont complets ou quasi-complets. Dans les repaires d'Hyène, il n'est pas rare de découvrir des os courts ou des métapodes d'Ongulés complets ou quasi-complets (Fosse 1994 ; Letourneux 2003 ; Villa et al. 2004 ; Discamps 2011).

$20 \mathrm{Au}$ final, malgré les limites imposées à l'étude taphonomique par la mauvaise conservation des surfaces corticales (abrasion causée par un charriage à sec?), plusieurs éléments plaident en faveur de l'existence d'un repaire d'Hyène à Bourdette : abondance de l'Hyène, présence d'hyénons, abondance de coprolithes, os rongés, restes digérés, morphotypes caractéristiques sur certaines pièces, répartition squelettique des os complets. Les ensembles fauniques de Bourdette semblent ainsi correspondre à un palimpseste d'épisodes d'utilisation de la grotte comme tanière d'Ours et repaire d'Hyène, sans qu'aucune action anthropique n'ait été identifiée sur les ossements. Il ne semble pas exister de différences taphonomiques nettes entre les différents locus ou couches, la majorité des variations étant potentiellement imputable à la faiblesse numérique des échantillons. 
Figure 3 - Comparaison du degré de destruction des humérus de Rhinocéros entre $A)$ Bourdette (SCB II c. 7b) et B) Camiac (Discamps 2011). Vues postérieure (A-1, B-1) et antérieure (A-2, B-2).
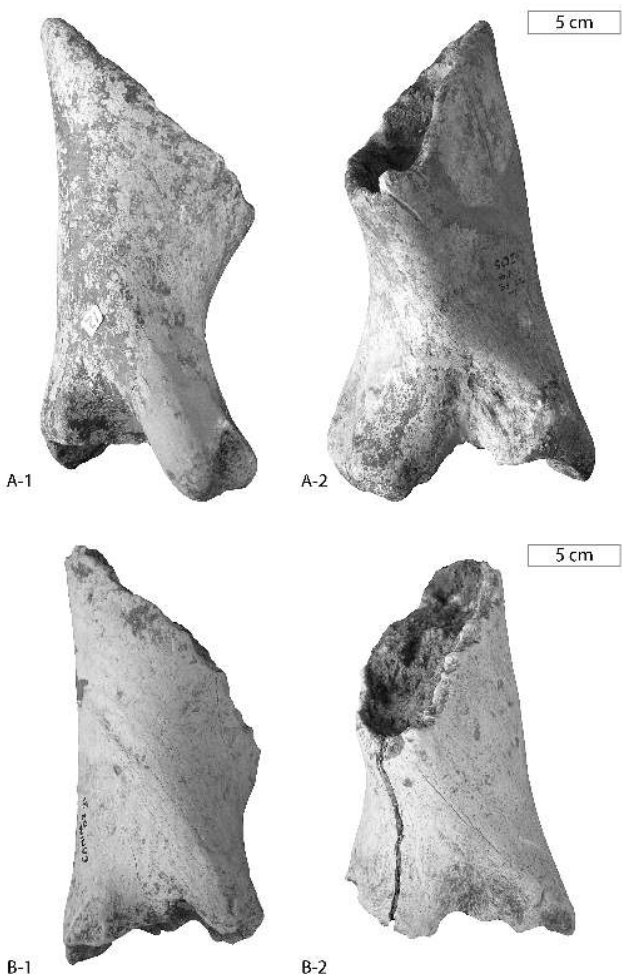

B-2

Tableau 5 - Abondance des os complets ou quasi complets par locus, couche et taxon (nombre total de restes squelettiques et pourcentages de restes concernés).

\begin{tabular}{|c|c|c|c|}
\hline $\begin{array}{c}\text { Tous } \\
\text { taxons }\end{array}$ & Bovinés & Cheval & Hyène \\
\hline
\end{tabular}

\begin{tabular}{|c|c|c|c|c|c|}
\hline \multirow{2}{*}{ Tous locus } & NR squel. & 247 & 60 & 63 & 73 \\
\cline { 2 - 6 } & $\%$ CO - ACO & $\mathbf{5 3} \%$ & $\mathbf{5 0} \%$ & $\mathbf{4 0} \%$ & $\mathbf{6 4} \%$ \\
\hline
\end{tabular}

\begin{tabular}{|c|c|c|c|c|c|}
\hline \multirow{2}{*}{ Gal. I; c. 8} & NR squel. & 38 & 20 & 10 & 3 \\
\cline { 2 - 5 } & $\%$ CO - ACO & $50 \%$ & $60 \%$ & $50 \%$ & $0 \%$ \\
\hline
\end{tabular}

\begin{tabular}{|c|c|c|c|c|c|}
\hline \multirow{2}{*}{ GaI. II ; c. 6} & NR squel. & 19 & 2 & 1 & 2 \\
\cline { 2 - 5 } & $\%$ CO - ACO & $58 \%$ & $0 \%$ & $0 \%$ & $0 \%$ \\
\hline
\end{tabular}

\begin{tabular}{|c|c|c|c|c|c|}
\hline \multirow{2}{*}{ Gal. II ; c. 7 } & NR squel. & 77 & 6 & 12 & 39 \\
\cline { 2 - 6 } & $\%$ CO - ACO & $68 \%$ & $67 \%$ & $50 \%$ & $79 \%$ \\
\hline
\end{tabular}

\begin{tabular}{|c|c|c|c|c|c|}
\hline \multirow{2}{*}{ Gal. II; c. 8 } & NR squel. & 53 & 13 & 17 & 16 \\
\cline { 2 - 6 } & $\%$ CO - ACO & $51 \%$ & $62 \%$ & $41 \%$ & $50 \%$ \\
\hline
\end{tabular}


Figure 4 - Industrie lithique de Bourdette ( $n^{\circ} 1-5,7$ et $9:$ SCB II; $n^{\circ} 6$ et $8:$ hors stratigraphie). $n^{\circ} 1$ : éclat tronqué et retouché passant au bec; $n^{\circ} 2:$ petit éclat retouché $; n^{\circ} 3:$ petit éclat levallois (quelques retouches et encoche $; n^{\circ} 4$ : éclat à retouche abrupte alterne; $n^{\circ} 5$ : nucléus en calcaire ; $n^{\circ} 6$ : enlèvements sur petit galet de quartz; $n^{\circ} 7$ : outil sur galet de quartz; $n^{\circ} 8:$ biface partiel à talon réservé sur galet de quartz; $\mathrm{n}^{\circ} 9$ : nucléus en quartzite.

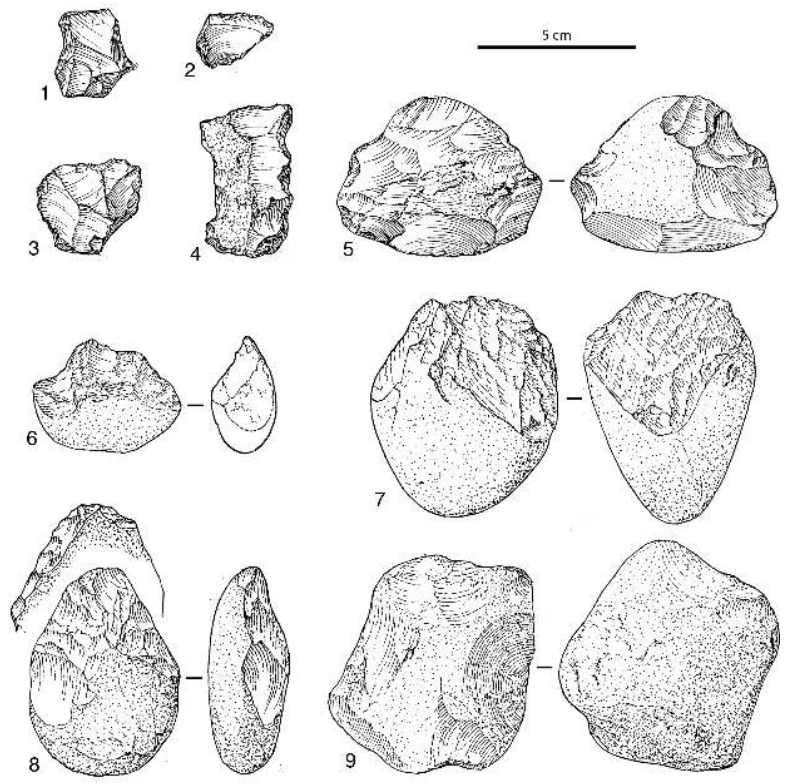

\section{4 - Vestiges lithiques : des occupations humaines ?}

21 L'industrie lithique de Bourdette, de petites dimensions dans son ensemble, est surtout faite à partir de petits galets de quartz ou de quartzite, plus rarement en chaille ou silex et elle comporte quelques éléments roulés.

\section{1 - Pièces recueillies en stratigraphie}

Dans la galerie I, seule la couche 8 a délivré des vestiges lithiques, dont deux éclats retouchés en silex et un en quartz, mais l'industrie reste très rare $(n=4)$.

L'industrie lithique est un peu plus abondante dans la deuxième galerie. Seulement trois pièces appartiennent à la couche 6 dont un éclat de quartz retouché ainsi qu'un éclat de silex noir roulé, à plages corticales et retouche abrupte alterne épaisse (fig. 4 , $\left.\mathrm{n}^{\circ} 4\right)$. Dans la couche 7 , il faut signaler parmi l'industrie $(\mathrm{n}=15)$ une petite pièce à retouche bifaciale, un petit grattoir sur fragment de galet de quartzite, trois microoutils sur galet, un polyèdre et un chopping-tool en quartz (fig. $4, n^{\circ} 7$ ). C'est dans la couche 8 que les vestiges lithiques sont les plus abondants $(n=21)$. Outre de petits éclats plus ou moins retouchés en silex ou en quartzite, ce niveau a livré un racloir sur éclat de quartz, trois petits outils sur galets de quartz et de quartzite, un bec sur éclat de silex (fig. $4, \mathrm{n}^{\circ} 1$ ), un éclat Levallois atypique en silex lacustre qui porte une encoche (fig. $4, \mathrm{n}^{\circ} 3$ ), un denticulé en quartz, un racloir en quartzite, un débris de quartz à 
micro-encoche, un galet de quartzite cassé à retouche abrupte-alterne ainsi que deux nucléus roulés, l'un en quartzite à grain fin (fig. 4, $n^{\circ} 9$ ) et l'autre en calcaire (fig. 4, $\mathrm{n}^{\circ} 5$ ). Une seule pièce provient de la couche 9 , un éclat de quartzite à retouche abruptealterne épaisse.

\section{2 - Pièces hors stratigraphie}

L'industrie lithique recueillie sur le site de Bourdette provient également en partie de ramassages en surface sur le plateau qui domine les cavités et en partie de déblais de carrière issus de la destruction partielle du remplissage.

La série recueillie en surface n'est pas très abondante $(n=27)$. Elle comporte des galets taillés unifaces ou bifaces, quelques éclats bruts et quelques éclats retouchés ainsi qu'un nucléus informe.

Parmi les pièces recueillies dans les déblais de carrière $(n=16)$, il y a un polyèdre en silex, un biface partiel à talon réservé sur galet de quartz (fig. $4, n^{\circ} 8$ ), six outils sur galet, deux pièces à encoche, un galet de quartz cassé et quelques éclats de quartzite.

\section{3 - Quelle place pour le lithique?}

27 Ces séries numériquement faibles mais relativement riches en outils et objets retouchés sont peu expressives et elles pourraient tout aussi bien être attribuées au Paléolithique ancien qu'au Paléolithique moyen. Il existe cependant d'étroites similitudes entre le matériel recueilli en surface sur le plateau et celui recueilli dans le remplissage des cavités.

L'ensemble apparait relativement homogène et il est fort probable que les vestiges recueillis en stratigraphie dans le remplissage des cavités soient en position secondaire, après avoir été entraînés à partir de lambeaux de dépôts alluviaux de surface qui nappaient le plateau. Cette hypothèse est appuyée par la présence de pièces roulées et la disposition de certains vestiges lithiques de la couche 8 du locus II, retrouvés en position verticale, redressés. Ce type de remaniements a été démontré dans d'autres sites paléontologiques à industrie lithique (Villa et Soressi 2000).

Figure 5 - Lignée Ursus. Typologie de l'entoconide de la carnassière inférieure (LM1) adaptée d'après Argant 1995. Les types C.1-2+ et C.2-2+, en grisés, ont été figurés de manière à représenter toute la variabilité des ours de Bourdette.

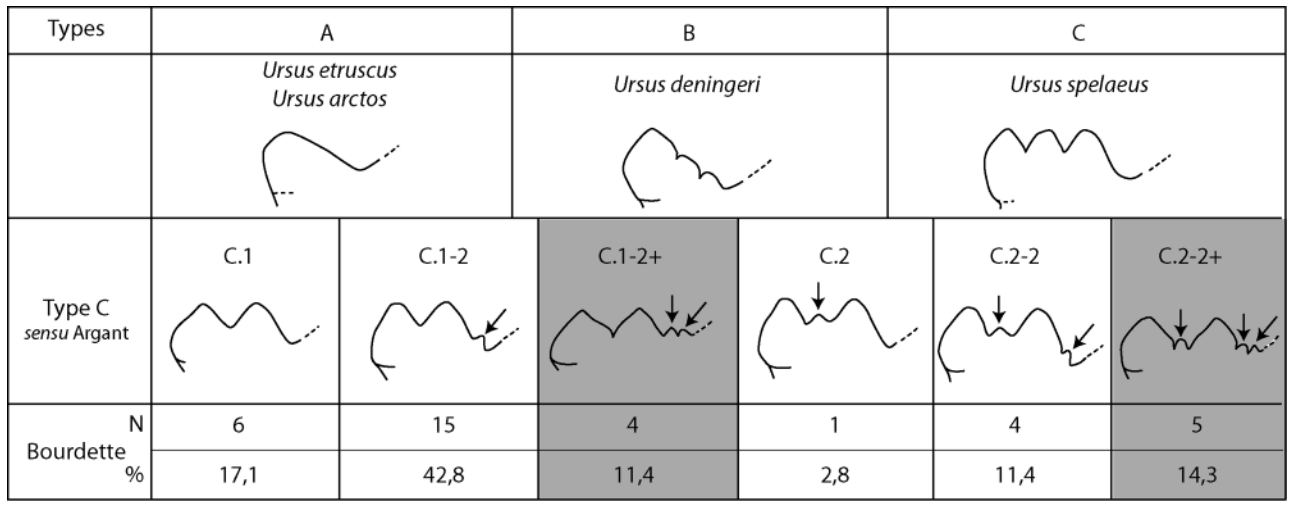


Figure 6 - Crocuta crocuta spelaea. Longueurs (moyennes et intervalles de confiance à $95 \%$ ) des carnassières inférieures $(M / 1)$ et quatrièmes prémolaires inférieures $(P / 4)$ à Bourdette en comparaison aux populations d'hyènes des Plumettes (Beauval 1997), d'Unikoté (Anselmo 2007), de Châteaubourg (Pautret-Homerville 2001), de Camiac (Guadelli 1987), de Gerde (Clot 1980), de Jaurens (Ballesio 1979), d'Arcy-sur-Cure (Baryshnikov 1999), de Tournal de Bize et Portel Ouest (Testu 2006), d'Artenac, Fontéchevade et Rochelot (Tournepiche 2009). Les ellipses de couleur délimitent de façon approximative l'intervalle de variation des formes tempérées (gris foncé) et froides (gris clair ; cf. Klein et Scott 1989 ; Tournepiche 2009).

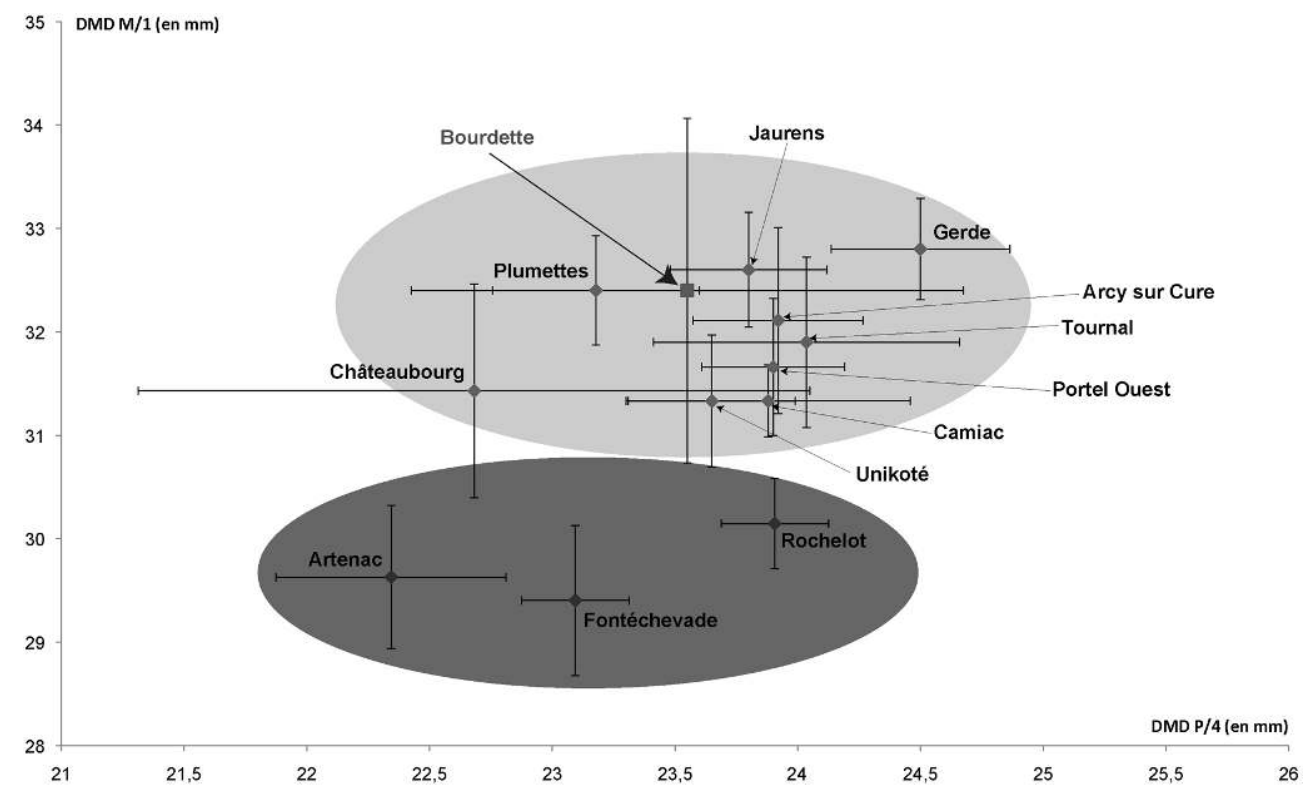

\section{5 - Nouvelles données chronologiques}

\section{1 - Données biochronologiques}

Le stade évolutif des taxons analysés fournit des informations intéressantes d'un point de vue biochronologique.

Pour l'Ours, les analyses menées sur 35 carnassières inférieures provenant des couches 7 et 8 de la deuxième galerie démontrent que nous sommes en présence d'Ours des cavernes (Ursus spelaeus) évolué (fig. 5). La présence de denticules surnuméraires sur l'entoconide de ces dents contribue à définir des types intermédiaires entre ceux définis par A. Argant (1995). Cette hyper-tuberculisation des carnassières inférieures couplée à la présence d'une crête distale sur les P4 inférieures (types E3-F3 de Rabeder 1992) démontre le caractère très évolué de ces ours.

Pour l'Hyène, les mesures effectuées sur les carnassières vont également dans le sens d'une accumulation des individus à la fin du Pléistocène (fig. 6). La forte taille des hyènes de Bourdette, dans le locus I comme dans le II, indiquerait une période froide compatible avec les données obtenues sur des hyènes du MIS 3, comme par exemple à Camiac (Guadelli 1987), aux Plumettes (Beauval 1997) ou à Jaurens (Ballésio 1979).

Seuls six restes de Loup ont été identifiés, tous dans le locus II. Parmi ces derniers, un humérus complet nous permet d'évaluer la stature des individus de Bourdette. La confrontation de ces mesures (fig. 7) avec celles obtenues sur des populations du sud de la France datées de la fin du Pléistocène moyen et du Pléistocène supérieur (Boudadi- 
Maligne 2010) permet de rapprocher le Loup de Bourdette de la population de l'aven de l'Arquet (c.a. $40 \mathrm{ka} \mathrm{BP}$ ).

En ce qui concerne le Cheval, d'un point de vue morphologique il ne fait pas de doute que nous avons affaire à un caballin au sens strict; qu'il s'agisse des dents ou du squelette post-crânien le Cheval de Bourdette, tous loci confondus, ne se différencie pas des caballins actuels. Nous ne reviendrons pas ici sur le débat byzantin d'une attribution des équidés caballins pléistocènes à Equus caballusou à Equus feruscar en l'absence d'arguments vraiment décisifs à l'appui du second nous continuerons à utiliser le nom d'Equus caballus et les sous-espèces définies dans le sud-ouest de l'Europe. Pour définir la sous-espèce représentée à Bourdette, nous sommes handicapés par le faible nombre de restes mesurables à notre disposition. Toutefois les quelques pièces mesurables indiquent Equus caballus gallicus dans une forme très proche de celle du site de Camiac (daté de 40-45 ka cal. BP ; cf. Discamps 2011), c'est-à-dire légèrement plus grande que la forme type du Gravettien de Solutré. Ceci étant, quelques pièces, malheureusement très érodées, semblent plus petites que les autres. Nous n'insisterons pas plus sur cette observation qui est peut-être visuellement guidée par le degré de corrosion, mais il ne faut pas non plus écarter d'emblée l'hypothèse que quelques pièces soient plus récentes.

En ce qui concerne Equus hydruntinus, même si le nombre de pièces mesurables est faible, le diamètre mésio-distal $\mathrm{du}$ protocône et l'indice protoconique des deux prémolaires mesurables indiquent la forme Equus hydruntinus davidi, qui est connue dans le quart sud-ouest de l'Europe à partir de 75-70 ka, et non à $E$. h. hydruntinus de Romanelli.

L'ensemble de ces données est extrêmement homogène et permet de dater l'accumulation de ces espèces à une phase froide de la fin du Pléistocène. L'association des espèces identifiées est relativement typique de ce qui est connu pour le début du MIS 3, à la fin du Moustérien et éventuellement au Châtelperronien, entre 60 et $40 \mathrm{ka}$ cal. BP, avec une abondance de bovinés et chevaux, une présence importante de l'Hyène et quelques restes de Rhinocéros laineux, Mammouth et Mégacéros (Guadelli 1987; Discamps et al. 2011 ; Discamps 2011). Les associations fauniques sont globalement similaires entre couches et entre locus.

Ces résultats ne sont pas en désaccord avec les hypothèses chronologiques déjà formulées sur la base des études sédimentologiques (Le Tensorer 1979, 1981) et palynologiques (Vivent 1988), mais les interprétations de ces études anciennes (bâties sur des découpages chronologiques et des méthodes aujourd'hui pour grande partie abandonnés) restent difficiles à intégrer aux résultats plus récents. 
Figure 7 - Canis lupus. Diamètre antéro-postérieur de la trochlée latérale de l'humérus en fonction de la largeur distale maximale pour différentes populations du Pléistocène moyen (COU I:

Coudoulous I) et supérieur (ARQ : aven de l'Arquet ; JAU : grotte de Jaurens ; MALD : grotte de Maldidier). Données de comparaison issues de Boudadi-Maligne 2010.

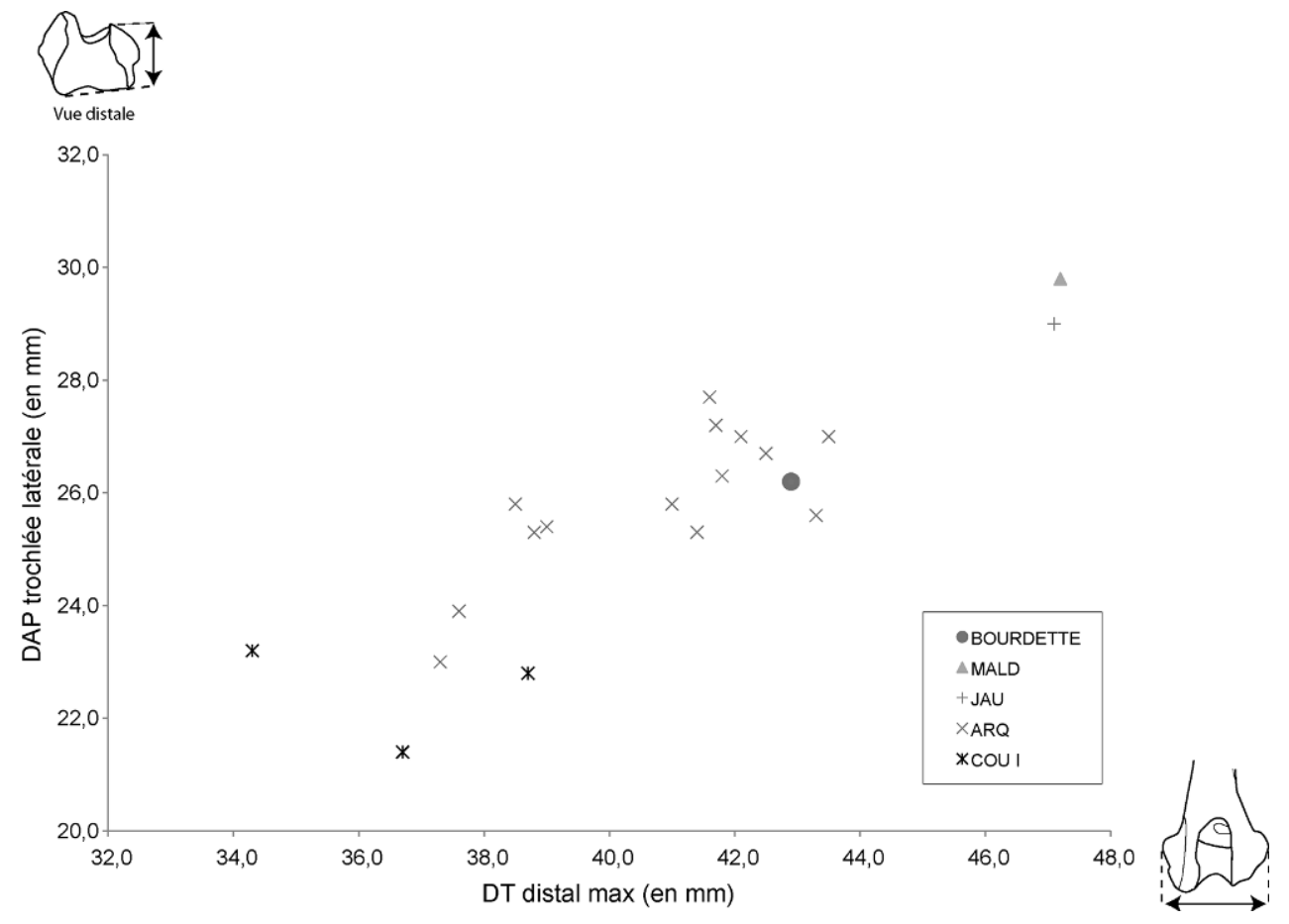

Figure 8 - Distributions calibrées des datations radiocarbone conventionnelles (gris clair) et AMS (gris foncé) disponibles pour les différents ensembles de Bourdette (courbe IntCal09; OxCal 4.1).

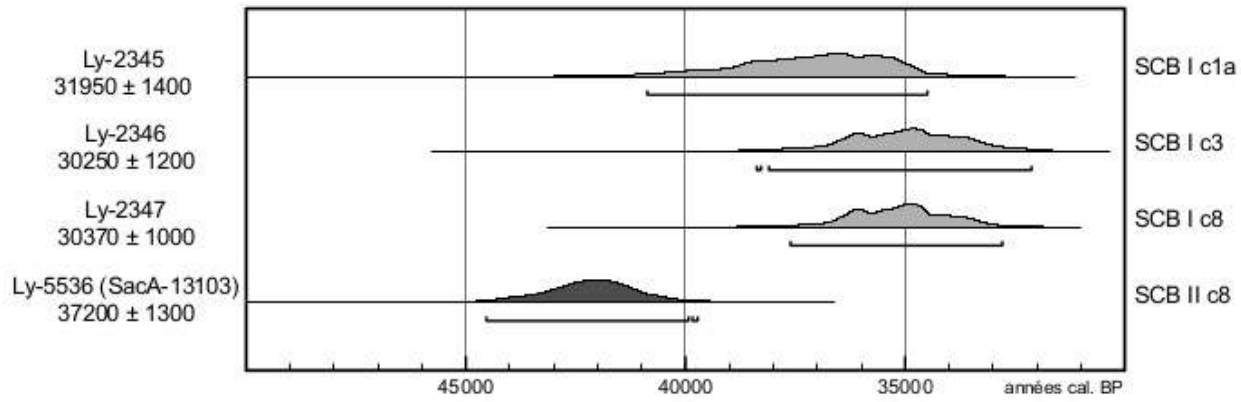


Figure 9 - Comparaison du degré de destruction des humérus d'Ours entre A) Bourdette (SCB II C. 7b) et B) La Chauverie (Discamps 2011). Vues antérieures.

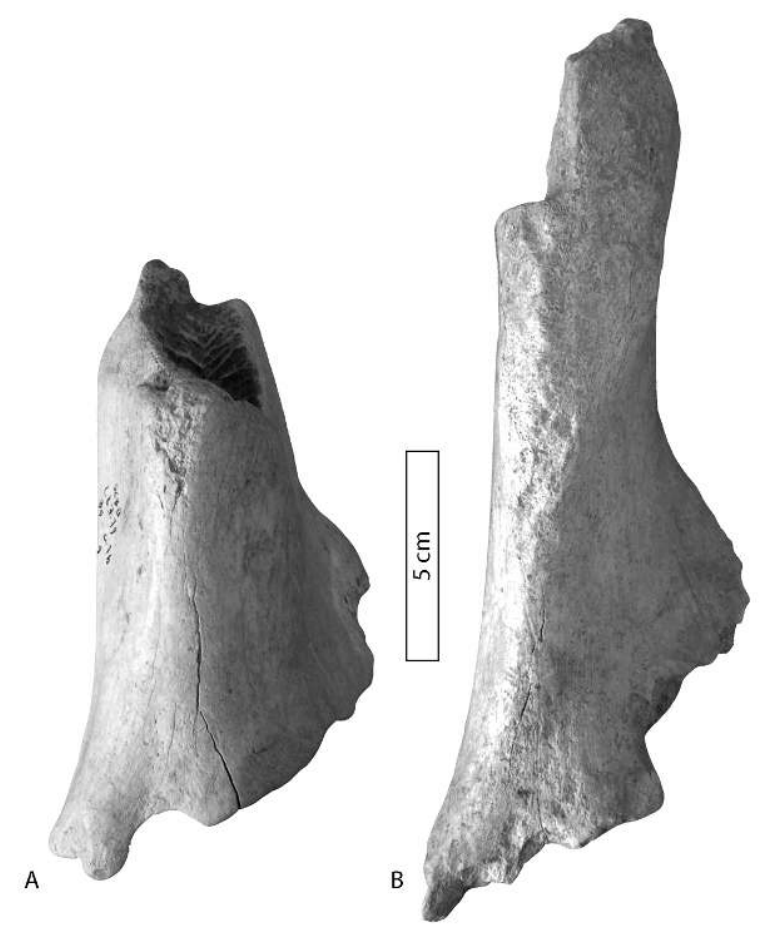

\section{2 - Datations absolues}

Trois datations radiocarbone conventionnelles avaient précédemment été réalisées sur le matériel du locus I de Bourdette, plaçant le gisement entre 32 et 41 ka cal. BP (Evin, inédit; fig. 8). Dans le cadre de ce travail, cinq échantillons ont été sélectionnés afin d'obtenir des datations AMS pour les différentes occupations du gisement : trois restes d'Ours, un d'Hyène et un de Cheval. Malheureusement, seul le reste de Cheval a pu être daté (Ly-5536 / SacA-13103 : $37200 \pm 1300$ BP), les autres échantillons présentant des taux de collagène résiduel trop faibles. Cette nouvelle datation est légèrement plus ancienne que celles précédemment obtenues, ce qui n'est pas étonnant au regard des progrès réalisés ces dernières décennies dans les procédures de traitement des échantillons ${ }^{14} \mathrm{C}$ (Evin 2004 ; Higham 2011). Ces progrès techniques peuvent à eux seuls expliquer les différences entre datations conventionnelles et AMS, et il serait prématuré de considérer que le locus I représente des occupations postérieures au locus II (si l'on considère les distributions de dates à deux écarts types, une des dates conventionnelles et la date AMS se recouvrent partiellement; cf. fig. 8). Au moins une partie des occupations de Bourdette serait donc à placer autour de 40-45 ka cal. BP, en accord avec les données biochronologiques (cf. 5.1), même si l'interprétation des datations radiocarbone obtenues pour ces périodes anciennes restent toujours problématiques (Higham 2011). 


\section{3 - Chronologie des occupations et arguments taphonomiques complémentaires} particulièrement délicat de discuter de la chronologie des occupations à Bourdette. Si l'on privilégie l'hypothèse du charriage à sec, l'abondance des os émoussés (cf. 3.2) pourrait plaider en faveur d'une occupation par les ours postérieure à celles des hyènes. Cependant, une partie des ossements d'Ours (non quantifiée dans ce travail) est également émoussée comme le reste de la faune. De plus, certains ossements d'Ours présentent des traces de consommation par les Carnivores. Quelques spécimens présentent des degrés de destruction tels qu'ils ne sont pas compatibles avec un cannibalisme intraspécifique, et indiquent donc qu'une partie des restes d'Ours a été consommée par les hyènes (fig. 9). Ce type d'éléments démontre qu'au moins une part des occupations de la grotte par l'Ours et par l'Hyène sont, à l'échelle géologique, contemporaines.

La grande majorité des données chronologiques provient de la galerie II (biochronologie de l'Ours et du Loup, majorité des restes d'Hyène, datation AMS) et il est donc difficile de statuer sur la chronologie des occupations selon les locus. L'homogénéité des données, chronologiques comme taphonomiques, permet cependant d'envisager qu'une bonne partie des occupations des différents locus sont potentiellement pseudo-contemporaines.

Les vestiges lithiques témoignent, eux, vraisemblablement d'occupations humaines du versant antérieures aux occupations animales de Bourdette, puisqu'ils semblent avoir été retrouvés en position remaniée. Leur attribution chronologique exacte (au Paléolithique ancien ou au Paléolithique moyen) reste délicate à établir.

\section{6 - Conclusion}

41 L'analyse interdisciplinaire menée à Bourdette a permis d'identifier les différents types d'occupations de la grotte. Ours et hyènes semblent s'être succédé, ces dernières ramenant avec elles les restes de certaines de leurs proies, Cheval et Bison en tête. L'Homme n'est lui présent qu'au travers de quelques vestiges lithiques qui semblent provenir du versant plutôt que de réelles occupations humaines de la grotte.

42 La chronologie exacte de chacune de ces fréquentations est particulièrement difficile à établir. L'ensemble des données chronologiques s'accorde pour placer la faune de Bourdette autour de $40 \mathrm{ka}$ cal. BP, au moins pour le locus II. Il reste délicat (voire impossible) de distinguer l'histoire exacte des successions d'occupations en l'absence de données spatiales. Ceci souligne l'intérêt des techniques de fouilles avec prise de coordonnées cartésiennes, y compris pour les sites paléontologiques (Discamps et al. sous presse). À Bourdette, la présence de restes d'Ours rongés par l'Hyène plaide cependant en faveur d'une contemporanéité (à l'échelle géologique) d'au moins une partie des tanières d'Ours et des repaires d'Hyène.

D'un point de vue méthodologique, la faune de Bourdette est riche d'enseignements. L'abondance des os émoussés a considérablement restreint l'étude taphonomique. Ce type de contexte met à mal les techniques classiques utilisées en taphonomie pour distinguer les agents accumulateurs. Bourdette rappelle que les discussions sur les 
agents accumulateurs d'ensembles fauniques ne peuvent pas se baser entièrement sur des pourcentages absolus (p.ex. d'os rongés ou de stries de découpe): certains processus naturels (vraisemblablement le charriage à sec dans le cas de Bourdette) peuvent induire à eux seuls la disparition des traces tant recherchées par les taphonomistes.

Nous tenons à remercier l'UMR PACEA 5199 au sein de laquelle cette recherche a été effectuée. Nous remercions également M.-F. Bonifay, S. Madelaine et le relecteur anonyme pour leurs différents commentaires.

\section{BIBLIOGRAPHIE}

ANDREWS P. 1995 - Experiments in taphonomy. Journal of Archaeological Science, 22, p. 147-153.

ANSELMO M. 2007 - Essai de caractérisation d'une population d'Hyènes des cavernes. Application au site d'Unikoté (Pyrénées-Atlantiques). Mémoire de Master 2, Université Bordeaux 1, 2007. 70 p.

ARGANT A. 1995 - Un essai de biochronologie à partir de l'évolution dentaire de l'ours des cavernes. Datation du site de la Balme à Collomb (Entremont-le-Vieux, Savoie, France). Quaternaire, 6, 3-4, p. 139-149.

BALLESIO R. 1979 - Le gisement pléistocène supérieur de la grotte de Jaurens à Nespouls, Corrèze, France. Les Carnivores (Mammalia, Carnivora). I : Canidae et Hyaenidae. Nouvelles Archives du Museum d'histoire naturelle de Lyon, 17, p. 25-55.

BARYSHNIKOV G. 1999 - Chronological and geographical variability of Crocuta spelaea (Carnivora, Hyaenidae) from the Pleistocene of Russia. Deinsea, 6, p. 155-173.

BEAUVAL C. 1997 - Le repaire d'Hyènes des Plumettes (Lussac-les-Châteaux, Vienne) : Etude paléontologique et observations taphonomiques. Mémoire de Maîtrise, Université Bordeaux 1, 1997. $121 \mathrm{p}$.

BEAUVAL C. et MORIN E. 2010 - Les repaires d'hyènes du Lussacois (Lussac-les-Châteaux, Vienne, France). Apport des sites des Plumettes et des Rochers de Villeneuve. In : BUISSON-CATIL J. et PRIMAULT J. (eds.), Préhistoire entre Vienne et Charente. Hommes et sociétés du Paléolithique. Chauvigny : Association des Publications Chauvinoises, p. 175-190.

BEHRENSMEYER A.K. 1990 - Transport-hydrodynamics : bones. In : Briggs D. E. G. et Crowther P. R. (eds.), Palaeobiology : a synthesis. Oxford : Blackwell Scientific Publications, p. 232-235.

BINFORD L. 1981 - Bones : ancient men and modern myths. Orlando : Academic Press.

BONA F. 2004 - Preliminary analysis on Ursus spelaeus Rosenmüller \& Heinroth, 1794 populations from « Caverna Generosa » (Lombardy - Italy). Cahiers scientifiques, Museum de Lyon, Hors série n², p. 87-98.

BOUDADI-MALIGNE M. 2010 - Les Canis pléistocènes du Sud de la France : approche biosystématique, évolutive et biochronologique. Thèse de Doctorat, Université Bordeaux 1, 2010. 451p. 
BOURDILLAT V. 2008 - Hommes - Carnivores? Caractériser l'action de l'hyène des cavernes : De l'utilisation des données fossiles pour l'interprétation des sites mixtes.Thèse de Doctorat, Muséum National d'Histoire Naturelle de Paris, 2008. 298 p.

BRAIN C.K. 1967 - Bone weathering and the problem of bone pseudo-tools. South African journal of Science, 63, p. 97-99.

BRUGAL J.-P., FOSSE P. et GUADELLI J.-L. 1997 - Comparative study of bone assemblages made by recent and Pleistocene Hyenids. In : HANNUS L. A., ROSSUM L. et WINHAM R. P. (eds.), Proceedings of the 1993 bone modification conference Hot Springs. South Dakota, p. 158-187.

BRUGAL J.-P. et JAUBERT J. 1991 - Les gisements paléontologiques pléistocènes à indices de fréquentation humaine : un nouveau type de comportement de prédation ? Paleo, 3, p. 15-41.

CAMPMAS É. et BEAUVAL C. 2008 - Consommation osseuse des carnivores : résultats de l'étude de l'exploitation de carcasses de boeufs (Bos taurus) par des loups captifs. Annales de paléontologie, 94, 3, p. $167-186$

CHAGNEAU J. 1980 - Sainte-Colombe-en-Bruilhos - Grotte de Bourdette. Gallia, 23, 2, p. 423-424.

CHAGNEAU J. 1982 - Sainte-Colombe-en-Bruilhois - Grotte de Bourdette. Gallia, 25, 2, p. 423-424.

CLOT A. 1980 - La grotte de la carrière (Gerde, Hautes-Pyrénées). Stratigraphie et péléontologie des Carnivores. Thèse de Doctorat, Toulouse, Université Paul Sabatier, 1980. 239 p.

COSTAMAGNO S. 1999 - Stratégies de Chasse et Fonction des Sites au Magdalénien dans le Sud de la France. Thèse de Doctorat, Université Bordeaux 1, 1999

COSTAMAGNO S., BEAUVAL C., LANGE-BADRÉ B., VANDERMEERSCH B., MANN A. et MAUREILLE B. 2005 - Homme ou carnivores? Protocole d'étude d'ensembles osseux mixtes : l'exemple du gisement moustérien des Pradelles (Marillac-le-Franc, Charente). Archaeofauna, 14, p. 43-68.

COUMONT M.-P. 2006 - Taphonomie Préhistorique : mammifêres fossiles en contexte naturel, les avenspièges, apport pour l'étude des archéofaunes. Thèse de Doctorat, Université Aix-Marseille I, 2006. $514 \mathrm{p}$.

COUMONT M.-P. 2009 - Proposition d'un référentiel taphonomique fossile de faunes issues d'avens-pièges. Annales de Paléontologie, 95, 1, p. 1-20.

CRUZ-URIBE K. 1991 - Distinguishing Hyena from Hominid bone accumulations. Journal of Field Archaeology, 8, p. 467-488.

DISCAMPS E. 2011 - Hommes et hyènes face aux recompositions des communautés d'Ongulés (MIS 5-3) : éléments pour un cadre paléoécologique des sociétés du Paléolithique moyen et supérieur ancien d'Europe de l'Ouest. Thèse de Doctorat, Université Bordeaux 1, 2011. 437 p.

DISCAMPS E., JAUBERT J. et BACHELLERIE F. 2011 - Human choices and environmental constraints : deciphering the variability of large game procurement from Mousterian to Aurignacian times (MIS 5-3) in southwestern France. Quaternary Science Reviews, 30, 19-20, p. $2755-2775$.

DISCAMPS E., DELAGNES, A., LENOIR, M. et TOURNEPICHE J.-F. sous presse. Interdisciplinary analysis (spatial, faunal and lithic data) as a tool for investigating Pleistocene sites with cooccurrences of humans and hyenas : the cases of Camiac and La Chauverie. Journal of Taphonomy, accepté.

EGELAND A.G., EGELAND C.P. et BUNN H.T. 2008 - Taphonomic Analysis of a Modern Spotted Hyena (Crocuta crocuta) Den From Nairobi, Kenya. Journal of Taphonomy, 6, 3-4, p. 275-299. 
ÉVIN J. 2004 - Les premières séries de datations radiocarbone concernant la Préhistoire française. In : Évin J. (ed.) Congrès du centenaire : Un siècle de construction du discours scientifique en Préhistoire. vol. 2, « Des idées d'hier... ». Paris : Mémoires de la Société Préhistorique Française, p. 87-95.

FAITH J.T. et BEHRENSMEYER A.K. 2006 - Changing patterns of carnivore modification in a landscape bone assemblage, Amboseli Park, Kenya. Journal of Archaeological Science, 33, 12, p. $1718-1733$.

FERNÁNDEZ-JALVO Y. et ANDREWS P. 2003 - Experimental effects of water abrasion on bone fragments. Journal of Taphonomy, 1, p. 147-163.

FOSSE P. 1994 - Taphonomie paléolithique : les grands mammifêres de Soleilhac (Haute-Loire) et de LunelViel 1 (Hérault). Thèse de Doctorat, Université Aix-Marseille I, 1994. 324 p.

FOSSE P. 1995 - Le rôle de l'Hyène dans la formation des assemblages osseux : 150 ans de controverses. L'apport des anciens textes de Préhistoire et de Paléontologie du Quaternaire aux études taphonomiques actuelles. Paleo, 7, p. 49-84.

FOSSE P. 1997 - Variabilité des assemblages osseux créés par l'Hyène des Cavernes. Paleo, 9, p. $15-54$.

FOSSE P., BRUGAL J.P., GUADELLI J.-L., MICHEL P. et TOURNEPICHE J.-F. 1998 - Les repaires d'hyènes des cavernes en Europe occidentale : présentation et comparaisons de quelques assemblages osseux. In : Brugal J.-P., Meignen L. et Patou-mathis M. (eds.), Économie préhistorique : les comportements de subsistance au Paléolithique. Antibes : Éditions APDCA, p. 43-62.

FOURVEL J.-B. 2008 - Etude paléontologique et taphonomique des vestiges osseux d'une tanière d'hyène tachetée fossile (Crocuta crocuta spelaea) du Pléistocène Supérieur : Conives (Indre, France). Mémoire de Master 2, Université de Toulouse II Le Mirail, 2008. 72 p.

GUADELLI J.-L. 1987 - Contribution à l'étude des zoocénoses préhistoriques en Aquitaine (Würm ancien et interstade würmien). Thèse de Doctorat, Université Bordeaux 1, 1987. 568 p.

HIGHAM T. 2011 - European Middle and Upper Palaeolithic radiocarbon dates are often older than they look : problems with previous dates and some remedies. Antiquity, 85, 327, p. 235-249.

KLEIN R.G. et SCOTT K. 1989 - Glacial/interglacial size variation in fossil spotted hyenas (Crocuta crocuta) from Britain. Quaternary Research, 32, p. 88-95.

KOBY F.E. 1943 - Les soi-disant instruments osseux du Paléolithique alpin et le charriage à sec des os d'ours des cavernes. Verhandlingen der Naturforschen der Gesellschaft in Basel, LIV, p. 59-95.

KOBY F.E. 1953 - Modifications que les ours des cavernes ont fait subir à leur habitat. InPremier Congrès International de Spéléologie, tome IV, section 4. Paris, p. 15-27.

KUHN B.F., BERGER L.R. et SKINNER J.D. 2010 - Examining criteria for identifying and differentiating fossil faunal assemblages accumulated by hyenas and hominins using extant hyenid accumulations. International Journal of Osteoarchaeology, 20, 1, p. 15-35.

LE TENSORER J.M. 1979 - Recherches sur le Quaternaire en Lot-et-Garonne : Stratigraphie, paléoclimatologie et préhistoire paléolithique. Thèse de Doctorat, Université Bordeaux I, 1979.

LE TENSORER J.-M. 1981 - Le Paléolithique de l'Agenais. Cahiers du Quaternaire nº 3.

LETOURNEUX C. 2003 - Devine qui est venu dîner à Brassempouy? Approche taphonomique pour une interprétation archéozoologique des vestiges osseux de l'Aurignacien ancien de la grotte des Hyènes (Brassempouy, Landes). Thèse de Doctorat, Université Paris 1, 2003. 319 p. 
MALLYE J.-B. 2007 - Les restes de Blaireau en contexte archéologique : Taphonomie, archéozoologie et éléments de discussion des séquences préhistoriques. Thèse de Doctorat, Université Bordeaux 1, 2007.

MAREAN C.W. et KIM S.Y. 1998 - Mousterian Large Mammal Remains from Kobeh Cave Behavioral Implications for Neanderthals and Early Modern Humans. Current Anthropology, 39, S1, p. S79-

S114.

MARRA A.C., VILLA P., BEAUVAL C., BONFIGLIO L. et GOLDBERG P. 2004 - Same predator, variable prey : taphonomy of two Upper Pleistocene hyena dens in Sicily and SW France. Revue de Paléobiologie, Genève, 23, 2, p. 787-801.

MOREL P. 1986 - Quelques polis naturels d'apparence trompeuse. Artéfacts, 3, p. 43-45.

PAUTRET-HOMERVILLE C. 2001 - Etude de l'Hyène des cavernes (Crocuta crocuta spelaea) en Ardèche. Origine, évolution et extinction d'un carnivore Pléistocène. Mémoire de Master 2, Muséum National d'Histoire Naturelle de Paris, 2001.

PÉREZ-RAMA M., GARCÍA-VÁZQUEZ A. et GRANDAL-D'ANGLADE A. 2010 - Acción de carnívoros sobre huesos de Ursus spelaeus (Rosenmüller) : carroñeo, canibalismo y explicaciones alternativas. In : BAQUEDANO E. et ROSELL J. (eds.), Actas de la $1^{a}$ Reunión de científicos sobre cubiles de hiena (y otros grandes carnívoros) en los yacimientos arqueológicos de la Península Ibérica. Madrid : Zona arqueológica, p. 518-523.

PICKERING T.R. 2002 - Reconsideration of criteria for differentiating faunal assemblages accumulated by hyenas and hominids. International Journal of Osteoarchaeology, 12, p. 127-141.

POKINES J.T. et KERBIS PETERHANS J.C. 2007 - Spotted hyena (Crocuta crocuta) den use and taphonomy in the Masai Mara National Reserve, Kenya. Journal of Archaeological Science, 34, 11, p. 1914-1931.

PRAT F. 1968 - Recherches sur les équidés pléistocènes en France. Thèse de Doctorat d'Etat, Université de Bordeaux, 1968. $662 \mathrm{p}$.

RABEDER G. 1991 - Die Höhlenbären der Conturines. Bozen : Athesia verlag, 124 p.

RABEDER G. 1992 - Das Evolutionsniveau des Höhlenbären aus dem Nixloch bei LosensteinTernberg. Mitteilungen der Kommission für Quartärforschung, 8, p. 133-141.

RENDU W. 2010 - Hunting behavior and Neanderthal adaptability in the Late Pleistocene site of Pech-de-l'Azé I. Journal of Archaeological Science, 37, p. 1798-1810.

RUITER D.J. (de) et BERGER L.R. 2000 - Leopards as Taphonomic Agents in Dolomitic Caves. Implications for Bone Accumulations in the Hominid-bearing Deposits of South-Africa. Journal of Archaeological Science, 27, 8, p. 665-684.

SALADIÉ P., HUGUET R., DÍEZ C., RODRÍGUEZ-HIDALGO A. et CARBONELL E. 2011 - Taphonomic modifications produced by modern brown bears (Ursus arctos). International Journal of Osteoarchaeology, accessible en ligne.

SHIPMAN P. et ROSE J. 1983 - Early hominid hunting, butchering and carcass processing behavior : approaches to the fossil record. Journal of Anthropological Archaeology, 2, p. 57-98.

STINER M.C. 1997 - Why do bear bones and artifacts co-occur in Paleolithic cave sites ? Observations from the Mediterranean rim. In : Tillet T. et Binford L. R. (eds.), L'Homme et l'ours / Man and bear. Colloque international / International Meeting, Auberives-en-Royans (Isère) du 4 au 6 novembre 1997, Grenoble. p. 1-23. 
STINER M.C., MUNRO N.D. et SANZ M. 2012 - Carcass damage and digested bone from mountain lions (Felis concolor) : implications for carcass persistence on landscapes as a function of prey age. Journal of Archaeological Science, 39, 4, p. 896-907.

TESTU A. 2006 - Étude paléontologique et biostratigraphique des Felidae et Hyaenidae pléistocènes de l'Europe méditerranéenne. Thèse de Doctorat, Université de Perpignan, 2006. 359 p.

TOURNEPICHE J.-F. 2009 - The fauna from Henri Martin's excavation of Bed E. In : Chase P. G., DebénatH A., Dibble H. L. et MCpherron S. P. (eds.), The Cave of Fontéchevade : Recent Excavations and their Paleoanthropological Implications. New York : Cambridge University Press, p. 139-153.

VILLA P., CASTEL J.-C., BEAUVAL C., BOURDILLAT V. et GOLDBERG P. 2004 - Human and carnivore sites in the European Middle and Upper Paleolithic : similarities and differences in bone modification and fragmentation. Revue de Paléobiologie, Genève, 23, 2, p. 705-730.

VILLA P. et SORESSI M. 2000 - Stone tools in carnivore sites : the case of Bois Roche. Journal of Anthropological Research, 56, 2, p. 187-215.

VIVENT D. 1988 - Analyses polliniques de coprolithes d'hyènes provenant des gisements suivant : SaintHyppolythe (Puy-de-Dome), Châtelperron (Allier), La Chaise de Vouthon (Charente), Cenac (Gironde), Camiac (Gironde), Bourdette (Lot-et-Garonne). Mémoire de Maîtrise, Université Bordeaux 1, 1988.

VOORHIES M.R. 1969 - Taphonomy and population dynamics of an Early Pliocene vertebrate fauna, Knox County, Nebraska. Wyoming University Contributions in Geology, Special Paper 1, p. 1-69.

\section{RÉSUMÉS}

À côté d'abondants indices d'occupation par l'Ours des cavernes (ossements, griffades, bauges), la grotte de Bourdette a livré les restes d'une vingtaine d'autres espèces ainsi que quelques vestiges lithiques. Une telle association de vestiges est a priori incompatible avec un fonctionnement seul de la grotte comme tanière d'Ours. Pour parvenir à distinguer les différentes occupations de Bourdette, et pour en établir la chronologie, une approche interdisciplinaire est ici développée en incluant l'étude taphonomique des restes osseux, l'analyse typotechnologique des pièces lithiques et la confrontation des différents éléments de chronologie disponibles (biochronologie, datations radiocarbone, attribution chronoculturelle des industries lithiques). À Bourdette se sont succédé ours et hyènes qui ont utilisé la grotte comme tanière et repaire, les dernières y ayant ramené les ossements de leurs proies (essentiellement bovinés et chevaux). Les vestiges lithiques semblent eux provenir de remaniements depuis le plateau, sans qu'aucune réelle occupation humaine du site ne puisse être démontrée. Les éléments de biochronologie et les datations radiocarbone disponibles s'accordent pour placer les occupations du site aux alentours de $40 \mathrm{ka}$ cal. BP. La présence d'ossements d'Ours rongés par les hyènes permet d'affirmer qu'au moins une partie des occupations de la grotte par les ours et les hyènes a été pseudocontemporaine. À Bourdette, les os sont particulièrement émoussés, possiblement à la suite des circulations ursines ("charriage à sec»): cette atteinte est si omniprésente qu'elle a considérablement restreint l'étude taphonomique, obligeant à reconsidérer les critères classiquement utilisés en taphonomie osseuse.

In Bourdette, if evidences of cave bear occupation are omnipresent (in the form of bones, claw marks and beds), the cave also delivered remains of more than a dozen other species as well as some lithic artifacts. Such a combination of different materials cannot be fully explained by the sole function of the cave as a bear den. In order to distinguish the different types of occupations in Bourdette and to establish their chronology, this paper proposes an interdisciplinary study 
that brings together data from faunal taphonomy, lithic typotechnology, biochronology and radiocarbon dating. Both Cave bear and Hyena used Bourdette as a den, the latter bringing remains of its preys in the cave (mostly Bovines and Horse). The presence of lithic artifacts in Bourdette seems only to be the result of post-depositional transport, so that men probably never occupied the cave themselves. Biochronological data and radiocarbon dates both date the deposits around $40 \mathrm{ka}$ cal. BP. Some bear bones were gnawed by hyenas, thus indicating that occupations of the cave by the two carnivores were, at least in part, broadly contemporaneous. The intensity of polishing is particularly intense on Bourdette bones, probably due to bear circulations in the cave ("charriage à sec"), to the point that procedures used commonly in bone taphonomy were severely undermined.

\section{INDEX}

Keywords : Taphonomy, Biochronology, Cave bear, Cave hyena, Upper Pleistocene, southwestern France

Mots-clés : Taphonomie, Biochronologie, Ours des cavernes, Hyène des cavernes, Pléistocène supérieur, sud-ouest de la France

\section{AUTEURS}

\section{EMMANUEL DISCAMPS}

Université Bordeaux 1, CNRS, PACEA, UMR 5199, 33405 Talence, France - Pour toute

correspondance : ediscamps@gmail.com

\section{MYRIAM BOUDADI-MALIGNE}

Université Bordeaux 1, CNRS, PACEA, UMR 5199, 33405 Talence, France

\section{JEAN CHAGNEAU}

Université Bordeaux 1, CNRS, PACEA, UMR 5199, 33405 Talence, France

DOMINIQUE ARMAND

Université Bordeaux 1, CNRS, PACEA, UMR 5199, 33405 Talence, France

JEAN-LUC GUADELLI

Université Bordeaux 1, CNRS, PACEA, UMR 5199, 33405 Talence, France

\section{MICHEL LENOIR}

Université Bordeaux 1, CNRS, PACEA, UMR 5199, 33405 Talence, France 